\title{
Focus on Vascular Function in Female Reproduction Regulation of vascular growth and function in the human placenta
}

\author{
G J Burton, D S Charnock-Jones ${ }^{1}$ and E Jauniaux ${ }^{2}$
}

Centre for Trophoblast Research and Department of Physiology, Development and Neuroscience, University of Cambridge, Downing Street, Cambridge CB2 3EG, UK, ${ }^{1}$ Centre for Trophoblast Research and Department of Obstetrics and Gynaecology, and the National Institute of Health Research Cambridge Biomedical Research Centre, University of Cambridge, Cambridge, CB2 2SW, UK and ${ }^{2}$ Academic Department of Obstetrics and Gynaecology, Institute for Women's Health, University College London, WC1E 6HX, London, UK

Correspondence should be addressed to G J Burton; Email: gjb2@cam.ac.uk

\begin{abstract}
During the course of 9 months, the human placenta develops into a highly vascular organ. Vasculogenesis starts during the third week post-conception. Hemangioblastic cell cords differentiate in situ from mesenchymal cells in the villous cores, most probably under the influence of vascular endothelial growth factor (VEGFA) secreted by the overlying trophoblast. The cords elongate through proliferation and cell recruitment, and connect with the vasculature of the developing fetus. A feto-placental circulation starts around 8 weeks of gestation. Elongation of the capillaries outstrips that of the containing villi, leading to looping of the vessels. The obtrusion of both capillary loops and new sprouts results in the formation of terminal villi. Branching and non-branching angiogenesis therefore play key roles in villous morphogenesis throughout pregnancy. Maternal circulating levels of VEGFA and placental growth factor vary across normal pregnancy, and in complicated pregnancies. Determining the impact of these changes on placental angiogenesis is difficult, as the relationship between levels of factors in the maternal circulation and their effects on fetal vessels within the placenta remains unclear. Furthermore, the trophoblast secretes large quantities of soluble receptors capable of binding both growth factors, influencing their bioavailability. Villous endothelial cells are prone to oxidative stress, which activates the apoptotic cascade. Oxidative stress associated with onset of the maternal circulation, and with incomplete conversion of the spiral arteries in pathological pregnancies, plays an important role in sculpting the villous tree. Suppression of placental angiogenesis results in impoverished development of the placenta, leading ultimately to fetal growth restriction.

Reproduction (2009) 138 895-902
\end{abstract}

\section{Introduction}

The human placenta is a highly vascular organ. By the end of gestation, it has developed a capillary network that is $\sim 550 \mathrm{~km}$ in length and $15 \mathrm{~m}^{2}$ in surface area (Burton \& Jauniaux 1995). This network is essential for effective materno-fetal exchange, but also plays a key mechanistic role in the elaboration of the placental villous tree. Vasculogenesis and subsequent angiogenesis are therefore of pivotal importance in placental development, and it is imperative that they are appropriately regulated. Failure to do so can lead to intrauterine fetal growth restriction and poor obstetric outcome.

This paper is one of four papers that form part of a special Focus Issue section on Vascular Function in Female Reproduction. The Guest Editor for this section was $\mathrm{HN}$ Jabbour, Edinburgh, UK.

\section{Morphological aspects of vascular development}

The first morphological evidence of vasculogenesis can be seen within the cores of mesenchymal villi at 18-20 days post-conception (pc), or at the end of the fourth week after the last menstrual period (LMP). Hemangioblastic cells differentiate in situ and form small clumps or cords of cells lying just beneath the trophoblastic epithelium. These precursor endothelial cells are united by either desmosomes or tight junctions, and the cords gradually acquire lumens and unite to form vessels (Dempsey 1972, Demir et al. 1989). The first signs of lumen formation are seen around 23 days pc, and immunohistochemical and morphological evidences suggest that apoptosis is involved in the process (Tertemiz et al. 2005). Early erythrocytes, still containing a nucleus, are often seen within the newly formed lumen, having differentiated from the inner aspect of the hemangioblastic cluster. The cords elongate through a 
combination of cell replication and recruitment of stromal cells, and ultimately connect with the vessels developing alongside the allantoic diverticulum in the connecting stalk, so establishing the feto-placental network. From the earliest stages, stromal cells are recruited as pericytes, and the percentage of capillary profiles associated with pericytes increases with gestational age (Zhang et al. 2002). A basement membrane, composed largely of type IV collagen, laminin, and fibronectin, does not develop until the third trimester, but ultimately surrounds both the endothelial cells and pericytes (Demir et al. 1989).

The endothelial cells of the human placenta are of the non-fenestrated type, and adjacent cells are linked by junctional complexes comprising both tight and adherens junctions (Heinrich et al. 1976, Jones \& Fox 1991). Besides stabilizing the capillary wall, these complexes regulate paracellular solute transport. Molecular studies have revealed that during the first trimester the tight junctions lack occludin and claudin- 1 and -2 , whereas the adherens junctions lack plakoglobin, molecules that are typically associated with mature forms of the respective junctions (Leach et al. 2002). This immaturity suggests that at this stage of gestation the capillaries are in a plastic state, suitable for remodeling, and are also highly permeable.

By 4 weeks pc (6 weeks post-LMP), capillary profiles can be observed peripherally in the villous core in close proximity to the trophoblast, along with some larger vessel profiles in the central region (Fig. 1). By now the capillaries are connected via the developing umbilical cord to the fetal heart, and to the vascular plexus of the yolk sac. The fetal heart has been beating for $\sim 1$ week

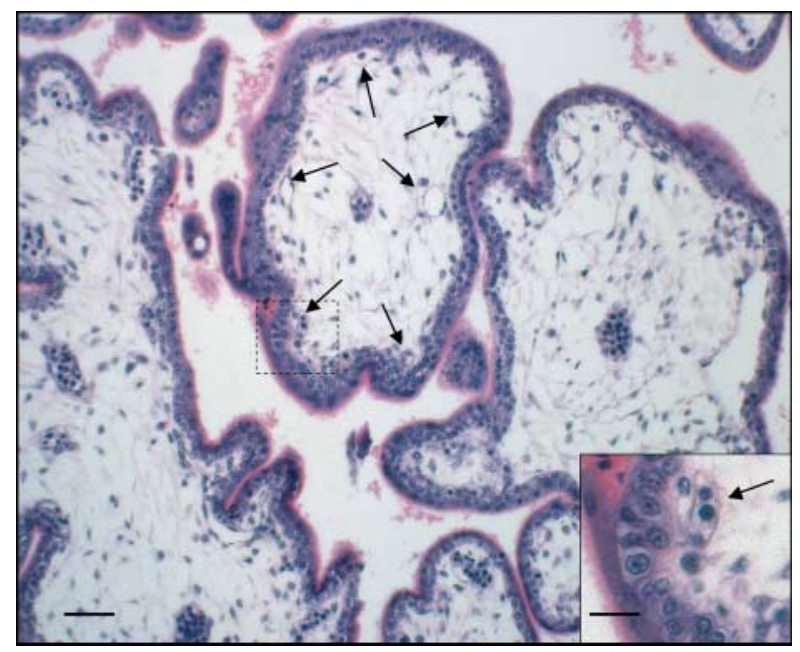

Figure 1 Photomicrograph of a villus from the central region of a placenta at 28 days pc showing a number of peripheral capillaries (arrowed) developing close to the trophoblastic epithelium, and some more centrally placed vessels. Note the presence of nucleated erythrocytes within the lumen of some capillaries. Inset shows one developing capillary containing two nucleated erythrocytes in greater detail. Scale bars, 50 and $15 \mu \mathrm{m}$ for inset. (from day 35 post-LMP), but an effective villous circulation is not established for a further 2 weeks (Jauniaux et al. 1991b). The delay in achieving flow appears to be due to the fact that most of the erythrocytes present within the early villous capillaries are nucleated, and so not readily deformable. They therefore impose a high resistance on the circulation, and this is reflected in the raised pulsatility index of the umbilical arterial waveforms at this stage of pregnancy. At the end of the first trimester, the resistance falls as the proportion of nucleated erythrocytes decreases rapidly.

The placental vascular network undergoes extensive and continual remodeling during pregnancy. During the first and early second trimesters, there is a gradual increase in the number, volume, and surface area of the capillary profiles within placental villi (Jauniaux et al. 1991 a, te Velde et al. 1997). The rate of increase in length accelerates at around 25 weeks, and total length increases exponentially until term (Fig. 2a; Mayhew 2002). The elongation is achieved through both proliferation of endothelial cells and their remodeling, with the surface area of each cell increasing towards term. The increase in capillary length outstrips that of the containing villi throughout pregnancy, so that from the start of the second trimester onwards the capillary/villus length ratio is constant at $\sim 3-4$ (Fig. 2b), although there is wide variation between individual placentas (Mayhew 2002). Indeed, disproportionate growth of the capillaries has long been thought to lead to the obtrusion of capillary loops from the villous surface, raising the trophoblastic epithelium in a blister-like fashion and initiating the formation of a new terminal villus (Kaufmann et al. 1985, 2004). The acceleration in capillary growth seen at around 25 weeks correlates closely with the formation of terminal villi in the same placental samples (Jackson et al. 1992), and so provides indirect support for this theory. However, estimation of capillary length using stereological techniques cannot distinguish between capillary elongation, with subsequent looping, and sprouting, for it is based only on counts of capillary profiles. Recently, three-dimensional reconstructions of images captured by confocal microscopy have revealed terminal villi forming in mature placentas by the obtrusion of both capillary loops and blind-ending (a)

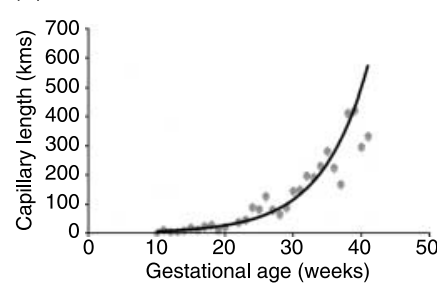

(b)

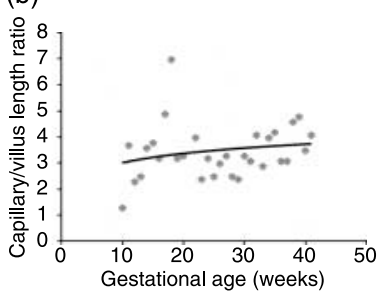

Figure 2 Scatter plots showing (a) total capillary length and (b) capillary/villus length ratio across gestation. For (a) an exponential coefficient provides the best line of fit, whereas for (b) it is a logarithmic relationship. Data are plotted from values given in Mayhew (2002). 


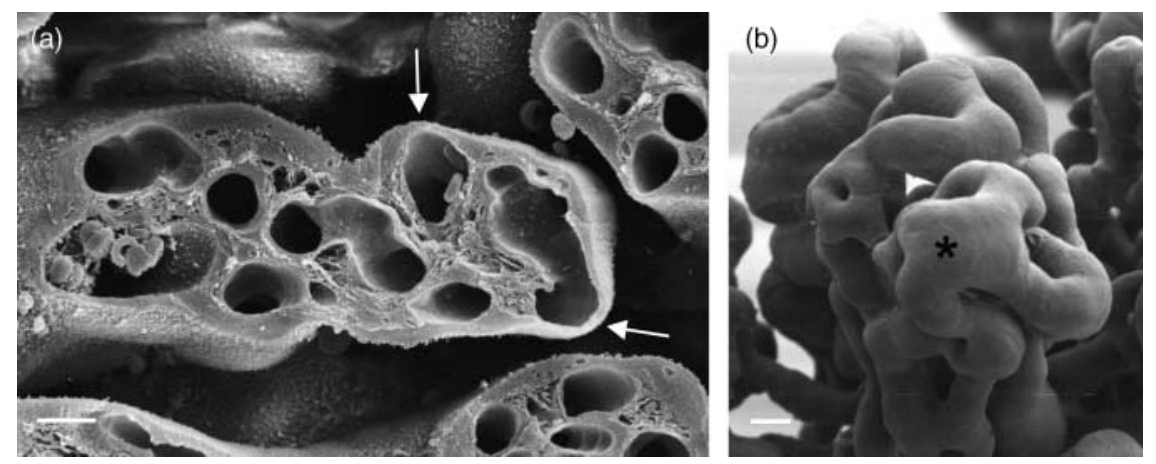

Figure 3 Scanning electron micrographs of (a) a freeze-cracked terminal villus and (b) a microvascular resin cast, from a mature placenta. In (a), the high degree of vascularity of a terminal villus is easily appreciated, and it can be seen that at points the capillaries are closely approximated to the villous covering (arrowed), forming vasculosyncytial membranes that are important for diffusional exchange. In (b), a dilated capillary sinusoid (asterisked) is seen, with at least three capillaries connecting to it. Scale bars, $10 \mu \mathrm{m}$.

sprouts (Jirkovska et al. 2008). It is now clear that at least two mechanisms for terminal villus formation operate, but each has angiogenesis as its driving force.

While some terminal villi contain only a simple capillary loop, other larger examples possess a more complex interconnecting network (Fig. 3a; Kaufmann et al. 1988, Jirkovska et al. 2008). Because of the relative sizes of the containing villi it is tempting to view this as a maturational process, and continuing angiogenic remodeling may serve to connect the arterio-venous circuit to that in the parent intermediate villus in a more efficient fashion. The observation that the junctional complexes linking adjacent endothelial cells within terminal villi demonstrate a molecular phenotype similar to that seen in the first trimester, suggesting they are relatively immature and plastic compared to the larger vessels of the villous tree, is consistent with this hypothesis (Leach et al. 2000, Leach 2002).

When viewing three-dimensional reconstructions or microvascular casts, it readily becomes apparent that the diameter of the capillaries is not constant along their length, but that localized dilations, referred to as sinusoids, occur. These are particularly prominent at the sites of acute bends, and they bring the outer wall of the capillary into close approximation to the trophoblast basement membrane (Fig. 3). Pressure exerted by the distended capillary is thought to lead to local thinning of the trophoblastic epithelium separating the maternal and fetal circulations, creating specialized areas for diffusional exchange. The importance of mechanical forces in sculpting vasculosyncytial membranes is emphasized by the fact that the thickness of the villous membrane at these sites is related to the hydraulic pressure differential between the maternal and fetal circulations (Burton \& Tham 1992).

\section{Molecular regulation of vasculogenesis and angiogenesis in the human placenta}

The molecular regulation of placental vasculogenesis and angiogenesis has been the subject of several recent exhaustive reviews (Charnock-Jones \& Burton 2000,
Leach et al. 2002, Charnock Jones et al. 2004, Demir et al. 2004, 2007, Benirschke et al. 2006). These reviews should be consulted for detailed descriptions of the various growth factors implicated, and their temporal and spatial localization. The following is a succinct synopsis of the principal regulators to indicate how changes in the intrauterine environment can influence villous vascularization at the molecular level, and hence placental function.

\section{Vascular endothelial growth factor}

Vascular endothelial growth factor (VEGFA) is considered to be the most important factor promoting the differentiation of mesenchymal cells in the villous core into hemangioblastic stem cells. It acts via two receptors, FLT1 and KDR. VEGFA is expressed intensely by the cytotrophoblast cells in the first few weeks of pregnancy, whereas the hemangiogenic cell cords show the strongest immunoreactivity for KDR. Mice in which VEGFA, or KDR, is knocked-out fail to initiate vasculogenesis (Shalaby et al. 1995, Carmeliet et al. 1996, Ferrara et al. 1996). Paracrine signaling may thus explain the proximity of the hemoangioblastic clusters to the trophoblastic epithelium. Later, expression of VEGFA in the cytotrophoblast cells wanes, whereas the villous macrophages and mesenchymal cells become strongly immunopositive. VEGFA is a powerful endothelial cell mitogen, and this switch may support angiogenic remodeling of the early vessels, stimulating the formation of a capillary network within the mesenchymal villus core (Demir et al. 2004).

\section{Placental growth factor}

The VEGF family also contains placental growth factor (PGF, also called PIGF), which, as its name suggests, is highly expressed in the trophoblast. Its function is less clear than that of VEGFA. PGF binds to FLT1 but not $\mathrm{KDR}$, and so may influence angiogenesis rather than vasculogenesis. However, PGF and FLT1 play a role in the mobilization of mesenchymal endothelial precursor 
cells that contribute to vasculogenesis (Li et al. 2006). In the past it has been viewed as a competitive inhibitor of VEGFA, for studies suggested it is a relatively weak mitogen for endothelial cells. However, in vivo data indicate that it may be as potent as VEGFA in stimulating new vessel growth (Ziche et al. 1997).

Longitudinal measurements of the placental concentrations of VEGFA and PGF throughout pregnancy are not possible, and so levels in the maternal plasma have been taken as a surrogate index of the placental angiogenic stimulus. This is complicated by the fact that the trophoblast secretes soluble receptors for both growth factors into the maternal circulation, influencing their bioavailability profoundly (Charnock Jones et al. 2004). Nonetheless, the general pattern observed is that maternal levels of total VEGFA rise gradually throughout pregnancy, whereas there is a marked increase in free PGF between 28 and 32 weeks of gestation. These profiles are altered in complicated pregnancies, for in cases of preeclampsia, maternal circulating levels of free PGF are suppressed.

\section{Angiopoietins}

The angiopoietins are another important family of growth factors that regulate angiogenesis in the placenta and elsewhere. Angiopoietin-1 and -2 are both ligands for the TIE2 tyrosine kinase receptor. ANG1-mediated phosphorylation of TIE2 promotes endothelial cell survival, and the recruitment of pericytes and smooth muscle cells that help to stabilize newly formed capillaries. In contrast, ANG2 is thought to act as a competitive inhibitor of ANG1, destabilizing the vessels and so rendering them more susceptible to the angiogenic stimulus of VEGFA or other growth factors. In the absence of a stimulatory growth factor, the vessels regress. ANG1 and 2 have been localized to the villous trophoblast from early gestation onwards, with RT-PCR data suggesting that the ratio of their mRNAs changes in favour of ANG1 as pregnancy advances (Charnock-Jones 2002, Seval et al. 2008). The TIE2 receptor is present on villous endothelial cells, and also the trophoblast, although its influence on trophoblast biology is unknown.

\section{Oxygen as a regulator of placental angiogenesis}

VEGFA, PGF, and the angiopoietins can all be regulated acutely by the local oxygen concentration, and so much attention has recently been focused on oxygen as a controller of placental development. VEGFA is regulated at the transcriptional level and also through mRNA stability, with low-oxygen concentrations stimulating expression in placental fibroblasts (Wheeler et al. 1995). Protein translation is also acutely regulated by the local oxygen concentration (Ray et al. 2009). PGF is controlled in a similar fashion but in the opposite direction, being suppressed at low levels of oxygen
(Ahmed et al. 2000). For the angiopoietins mechanisms differ; ANG1 is regulated by changes in mRNA stability, whereas ANG2 is regulated transcriptionally (Zhang et al. 2001). Consequently, under low oxygen, the ratio of ANG1:ANG2 shifts towards ANG2, favouring vessel instability, angiogenesis, and vessel remodeling.

It is now widely accepted that the human placenta develops under low-oxygen concentrations during the first trimester in the absence of a significant maternal circulation (Jauniaux et al. 2000), conditions that would be expected to support vasculogenesis and angiogenesis. In the absence of experimental manipulations, it is difficult to determine how critical this environment is, but there is indirect evidence that it may be crucial. First, increased numbers of hemangiogenic cords are present in early villi from pregnancies complicated by maternal anemia (Kadyrov et al. 1998), when the oxygen concentration in the developing placenta might be expected to be even lower than normal. Second, comparisons between villi located in the central and peripheral regions of the placenta indicate that hyperoxia has the opposite effect. Ultrasonography has shown that when maternal blood flow to the placenta commences at the end of the first trimester, it does so in a peripheral-central fashion (Jauniaux et al. 2003). Consequently, oxygen concentrations will be higher in the periphery, and increased levels of oxidative stress in villi sampled from this site provide supportive evidence that this is the case. These villi are notably either avascular or contain degenerate capillaries, consistent with the withdrawal of VEGFA support secondary to a hyperoxic environment (Alon et al. 1995). Similar changes are seen in placentas retained in utero in cases of missed miscarriage, when onset of the maternal circulation is premature and widespread throughout the placenta (Jauniaux et al. 2003). Again there is evidence of increased oxidative stress, particularly in the syncytiotrophoblast that is a prime source of VEGFA. There is a negative correlation between the volume of the fetal capillaries in the villi and the time for which the placenta has been retained after fetal death, indicating progressive regression of the capillary network under these hyperoxic conditions (Hempstock et al. 2003b).

At the end of the first trimester, oxygen concentrations within the placental intervillous space normally rise threefold (Jauniaux et al. 2000). Then, from 16 weeks they gradually fall, from $\sim 60$ to $40 \mathrm{mmHg}$ at term, as placental and fetal oxygen consumption increase (Soothill et al. 1986). The latter figures were obtained by aspiration of intervillous blood through the chorionic plate at the time of cordocentesis; and while providing an important guide as to placental oxygenation, they do not take into account regional variations in oxygen concentrations that may play an important role in regulating villus morphogenesis. Thus, when maternal blood is delivered into the placenta from the spiral arteries, it is directed to the center of a placental lobule, 
from which it then percolates peripherally. An oxygen gradient would therefore be expected across the lobule, but this cannot be confirmed by direct measurements using current technologies for practical and ethical reasons. However, measurements of antioxidant enzyme expression and activity in villous tissues sampled from different sites within lobules support this hypothesis (Hempstock et al. 2003a). It is also notable that dilated capillary sinusoids and vasculosyncytial membranes are more prominent in the peripheral regions of a lobule, where oxygen concentrations are predicted to be the lowest (Fox 1964, Critchley \& Burton 1987).

\section{Sprouting versus non-sprouting angiogenesis}

It has been postulated that there is a major switch in the pattern of angiogenesis within the placenta during gestation, with branching angiogenesis, the formation of new vessels through sprouting, occurring from day 32 pc to week 24, and non-branching angiogenesis, the formation of capillary loops through elongation, predominating thereafter to term (Kaufmann et al. 1985, 2004, Benirschke et al. 2006). This hypothesis is based on the assumption that the elaboration of terminal villi, which takes place in the second half of pregnancy, is driven by the obtrusion of capillary loops from the surface of intermediate villi as described previously. The protagonists have also alluded to the rise in maternal concentrations of PGF at mid-pregnancy to support their theory, suggesting that suppression of VEGFA activity by PGF inhibits capillary sprouting. Finally, they have linked this change in the angiogenic milieu to the rise in intraplacental oxygen concentrations associated with onset of the maternal circulation, although the data presented earlier indicate there is a lag phase of at least 2 months between the two events.

The evidence emerging from the three-dimensional reconstructions of Jirkovská et al. (2008) challenges this hypothesis, for they regularly observed terminal villi in mature placentas containing blind-ending capillary sprouts. The concept that branching angiogenesis continues beyond week 24 is supported indirectly by data from high-altitude pregnancies, in which the terminal villi are shorter and more clustered, suggesting increased capillary branching during the second half of pregnancy when the terminal villi are principally formed (Ali et al. 1996). Interestingly, the proportion of capillaries associated with pericytes is reduced at altitude compared with sea-level controls, conferring greater plasticity in a similar fashion as during the first trimester (Zhang et al. 2002). In addition, the finding that endothelial junctional complexes are immature in terminal villi again suggests these capillary networks are plastic even in late pregnancy (Leach 2002).

As discussed earlier, the bioavailability of these growth factors at the villous level during pregnancy is difficult to predict due to the secretion of soluble receptors. It is likely that the rate and mode of angiogenesis will not be dictated by one factor alone, but rather by a complex integration of signaling mechanisms of which oxygen and growth factors are two contributors. Indeed, a quantitative analysis of villous vascularization across the first to second trimester transition did not reveal any sudden changes in response to the rising oxygen levels (Jauniaux et al. 1991a). Although there may be changes in the pattern of placental angiogenesis during gestation, the hypothesis that there is a clear-cut dichotomous shift from branching to non-branching angiogenesis midpregnancy no longer appears tenable. Further studies quantifying capillary sprouting throughout gestation are required to resolve the issue.

\section{Mechanical factors in the regulation of placental angiogenesis}

By comparison with growth factors and oxygen, almost no attention has been paid to the role of mechanical forces in the regulation of placental angiogenesis. This is surprising given the wealth of data from other systems that they are powerful inducers of endothelial cell proliferation and motility (Shiu et al. 2005). Some evidence that may be important in the placenta is provided by the observation that the recruitment of perivascular cells as precursor smooth muscle cells, and the transformation into arteries and veins, is not observed until the start of the second trimester (Kaufmann et al. 2004), by which time a fetal circulation through the organ has been established.

Shear stress may be an important factor in the larger vessels of stem and intermediate villi, but at the level of terminal villi, the rate of flow is unlikely to be sufficient to generate significant forces. Cyclic strain is probably more important at these sites, especially given the acute changes in direction that must occur within the capillary plexus. This may explain the location of the dilated sinusoids at the apices of capillary loops (Burton \& Tham 1992). The observation that the capillary sinusoids are capable of expansion/compression dependent upon the pressure differential between the maternal and fetal circulations indicates that the capillary walls have elastic properties (Karimu \& Burton 1994). The pressure differential is likely to rise during gestation as the fetal heart matures, and so one might envisage a continually increasing distending force being applied. This will be resisted by the complement of intermediate fibres within the endothelial cells, and also by the composition of the extracellular matrix and the presence of encircling collagen fibres. The interaction between mechanical forces and local growth factors in sculpting vasculosyncytial membranes deserves further investigation, for these sites are of key importance to materno-fetal exchange. 

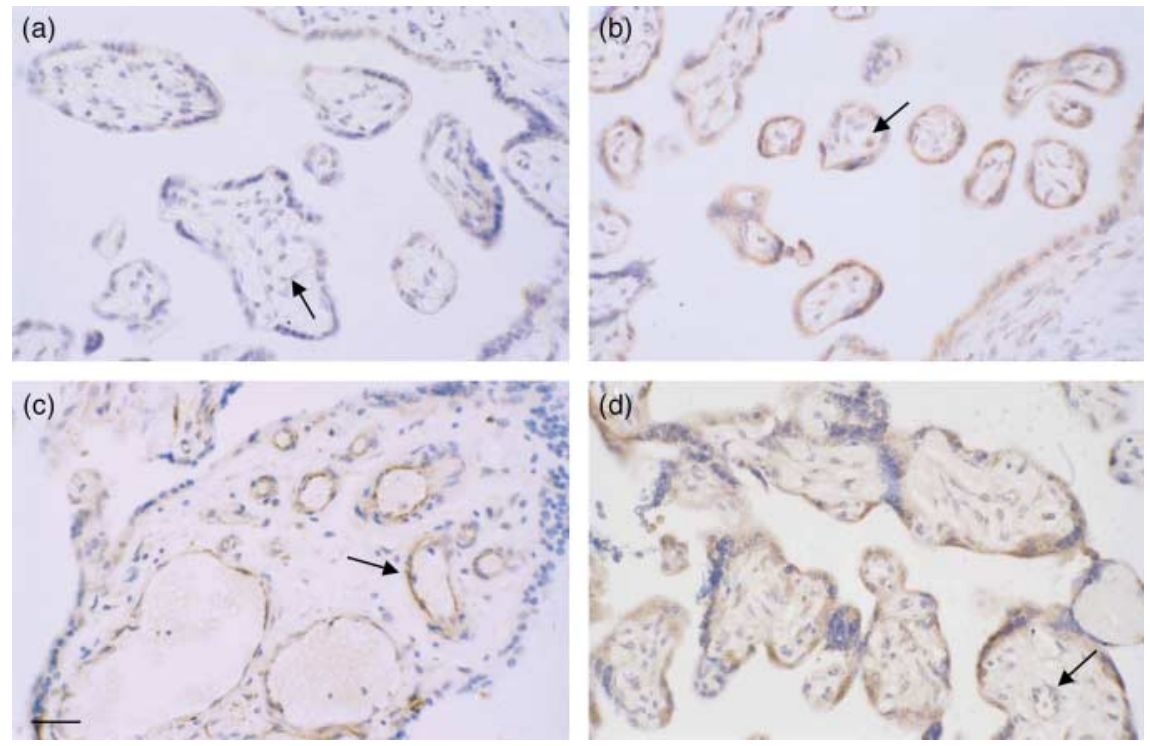

Figure 4 Immunohistochemistry for activated caspase- 3 in villi from mature placentas. (a) Normal non-labored caesarean delivery showing low levels of active caspase- 3 in the trophoblast and endothelial cells (arrowed), (b) a vaginally delivered placenta following labor showing strong immunoreactivity in the trophoblast and some endothelial cells (arrowed), (c) a non-labored caesarean-delivered placenta exposed to hypoxia-reoxygenation in vitro showing the intense activation of caspase- 3 in the endothelial cells of large and small fetal vessels (arrowed), (d) a non-labored caesarean-delivered placenta from a case of preeclampsia showing intense staining in the trophoblast, and some endothelial cells (arrowed). Scale bar, $50 \mu \mathrm{m}$ for all cases.

\section{Dysregulation of placental angiogenesis in pathological pregnancies}

Vascular development of the placenta is frequently perturbed in complications of pregnancy (Mayhew et al. 2004). Although the changes may be induced by several potential factors in the intrauterine environment, most attention has been focused on oxygen. Both placental hypoxia and hyperoxia have been implicated. Hypoxia, arising most often through compromise of the maternal arterial supply, is believed to result in exaggerated capillary and villous branching. These changes are most likely the result of subtle alterations in the ratios of VEGFA:PGF and ANG1:ANG2, but there is currently no firm evidence to confirm this hypothesis. Increased branching may increase placental efficiency by providing more arterio-venous circuits operating in parallel, returning fetal blood to the umbilical cord as soon as it has equilibrated with that in the intervillous space. However, excessive hypoxic stimulation of the endothelial cells can result in benign tumors termed chorioangiomas. In some forms of the tumor, large vessels develop and can lead to damaging arterio-venous shunts, whereas in others there is only disorganized endothelial hyperplasia. Chorioangiomas are more common at altitudes in excess of $4000 \mathrm{~m}$ (Reshetnikova et al. 1996), suggesting strongly that hypoxia plays an important role in their aetiology.

Hyperoxia is postulated to have the opposite effect, leading to reduced capillary branching and impoverished terminal villi (Kingdom \& Kaufmann 1997). It is argued that these changes will reduce oxygen extraction from the intervillous space, so promoting further hyperoxia. While this may be true it is a rather circular argument, and the initial precipitating cause of the placental vascular maldevelopment is not clear.
Hypoxia and hyperoxia are relative terms, and as discussed earlier the pattern of maternal blood flow through the lobule means that villi in different regions will experience different oxygen concentrations. The terms therefore have to be qualified according to the location and the stage of gestation that is being considered.

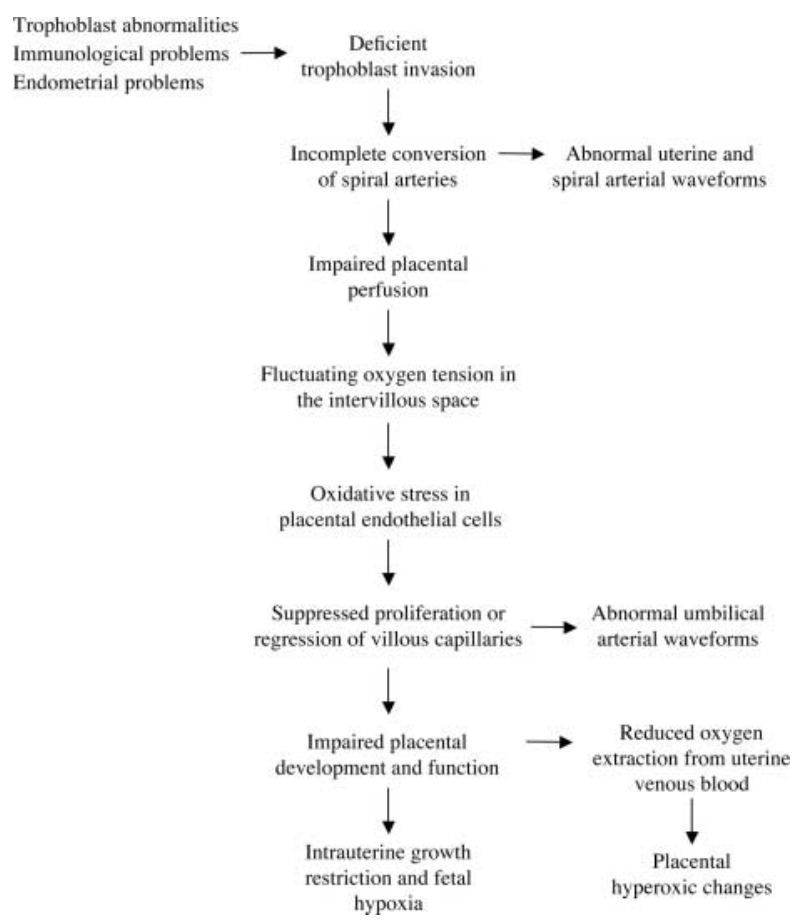

Figure 5 Flow diagram indicating how oxidative stress induced through deficient conversion of the spiral arteries may lead through impaired placental angiogenesis to fetal growth restriction. This may act as a final common pathway for various conditions that result in impaired trophoblast invasion (see Pijnenborg et al. (2006) for recent review). Adapted from Kuzmina et al. (2005) with permission. 
Our recent research indicates that the constancy of the prevailing oxygen concentration may be a more critical factor than the absolute value. We have hypothesized that the deficient conversion of the maternal spiral arteries associated with the majority of pregnancy complications increases the risk of spontaneous vasoconstriction of the arteries, and hence predisposes the placenta to ischemia-reperfusion-type injury. When normal placental explants are exposed to hypoxiareoxygenation in vitro, high levels of oxidative stress are generated in the trophoblast and endothelial cells, mimicking the changes seen in preeclampsia (Hung et al. 2001). We have observed the same distribution of oxidative stress in placentas from uncomplicated pregnancies following labour and vaginal delivery, while it is absent from those delivered by caesarean section (Fig. 4; Cindrova-Davies et al. 2007).

Oxidative stress is a powerful inducer of apoptosis, and we have observed activation of the executioner caspase- 3 in villous trophoblast and endothelial cells following labor and also hypoxia-reoxygenation in vitro (Fig. 4). We have therefore speculated that oxidative stress, secondary to defective spiral artery conversion, leads to arrest of placental angiogenesis, or even regression of existing capillaries (Fig. 5; Kuzmina et al. 2005). As a result, high-resistance waveforms become evident in the umbilical circulation, and there is associated fetal hypoxia and growth restriction due to the reduced diffusing capacity of the organ.

\section{Conclusion}

Vasculogenesis and angiogenesis are fundamental to the normal development and function of the human placenta. Details of the complex interplay of hypoxically regulated growth factors, their membrane-bound receptors and soluble receptors that controls the processes at the molecular level are beginning to be elucidated. In contrast, the role of mechanical stimuli is largely unknown, and deserves further research. Placental endothelial cells are highly prone to oxidative stress in vivo and in vitro, and this is likely to be a powerful negative regulator of angiogenesis in pathological pregnancies.

\section{Declaration of interest}

The authors declare they have no conflicting interests in the preparation of this review.

\section{Funding}

The authors gratefully acknowledge the support of the Wellcome Trust (069027/Z/02/Z and 084804/2/08/Z) for their research.

\section{References}

Ahmed A, Dunk C, Ahmad S \& Khaliq A 2000 Regulation of placental vascular endothelial growth factor (VEGF) and placenta growth factor (PIGF) and soluble Flt-1 by oxygen - a review. Placenta 21 S16-S24.

Ali KZM, Burton GJ, Morad N \& Ali ME 1996 Does hypercapillarization influence the branching pattern of terminal villi in the human placenta at high altitude? Placenta 17 677-682.

Alon T, Hemo I, Itin A, Pe'er J, Stone J \& Keshet E 1995 Vascular endothelial growth factor acts as a survival factor for newly formed retinal vessels and has implications for retinopathy of prematurity. Nature Medicine 1 1024-1028.

Benirschke K, Kaufmann P \& Baergen RN 2006 Pathology of the Human Placenta. New York: Springer.

Burton GJ \& Jauniaux E 1995 Sonographic, stereological and Doppler flow velocimetric assessments of placental maturity. British Journal of Obstetrics and Gynaecology 102 818-825.

Burton GJ \& Tham SW 1992 The formation of vasculo-syncytial membranes in the human placenta. Journal of Developmental Physiology 18 43-47.

Carmeliet P, Ferreira V, Breier G, Pollefeyt S, Kieckens L, Gertsenstein M, Fahrig M, Vandenhoeck A, Harpal K, Eberhardt C et al. 1996 Abnormal blood vessel development and lethality in embryos lacking a single VEGF allele. Nature 380 435-439.

Charnock-Jones D 2002 Soluble flt and the angiopoietins in the development and regulation of placental vasculature. Journal of Anatomy 200 607-615.

Charnock-Jones DS \& Burton GJ 2000 Placental vascular morphogenesis. Baillière's Best Practice \& Research. Clinical Obstetrics \& Gynaecology 14 953-968.

Charnock Jones DS, Kaufmann P \& Mayhew TM 2004 Aspects of human fetoplacental vasculogenesis and angiogenesis. I. Molecular recognition. Placenta 25 103-113.

Cindrova-Davies T, Yung HW, Johns J, Spasic-Boskovic O, Korolchuk S, Jauniaux E, Burton GJ \& Charnock-Jones DS 2007 Oxidative stress, gene expression, and protein changes induced in the human placenta during labor. American Journal of Pathology 171 1168-1179.

Critchley GR \& Burton GJ 1987 Intralobular variations in barrier thichkness in the mature human placenta. Placenta 8 185-194.

Demir R, Kaufmann P, Castellucci M, Erbengi T \& Kotowski A 1989 Fetal vasculogenesis and angiogenesis in human placental villi. Acta Anatomica 136 190-203.

Demir R, Kayisli UA, Seval Y, Celik-Ozenci C, Korgun ET, Demir-Weusten AY \& Huppertz B 2004 Sequential expression of VEGF and its receptors in human placental villi during very early pregnancy: differences between placental vasculogenesis and angiogenesis. Placenta 25 560-572.

Demir R, Seval Y \& Huppertz B 2007 Vasculogenesis and angiogenesis in the early human placenta. Acta Histochemica 109 257-265.

Dempsey EW 1972 The development of capillaries in the villi of early human placentas. American Journal of Anatomy 134 221-238.

Ferrara N, Carver-Moore K, Chen H, Dowd M, Lu L, O'Shea KS, PowellBraxton L, Hillan KJ \& Moore MW 1996 Heterozygous embryonic lethality induced by targeted inactivation of the VEGF gene. Nature $\mathbf{3 8}$ 239-442.

Fox H 1964 The pattern of villous variability in the normal placenta. Journal of Obstetrics and Gynaecology of the British Commonwealth 71 749-758.

Heinrich D, Metz J, Raviola E \& Forssmann WG 1976 Ultrastructure of perfusion-fixed fetal capillaries in the human placenta. Cell and Tissue Research 172 157-169.

Hempstock J, Bao Y-P, Bar-Issac M, Segaren N, Watson AL, Charnock Jones DS, Jauniaux E \& Burton GJ 2003a Intralobular differences in antioxidant enzyme expression and activity reflect oxygen gradients within the human placenta. Placenta 24 517-523.

Hempstock J, Jauniaux E, Greenwold N \& Burton GJ 2003b The contribution of placental oxidative stress to early pregnancy failure. Human Pathology 34 1265-1275.

Hung TH, Skepper JN \& Burton GJ 2001 In vitro ischemia-reperfusion injury in term human placenta as a model for oxidative stress in pathological pregnancies. American Journal of Pathology 159 1031-1043. 
Jackson MR, Mayhew TM \& Boyd PA 1992 Quantitative description of the elaboration and maturation of villi from 10 weeks of gestation to term. Placenta 13 357-370.

Jauniaux E, Burton GJ, Moscosco GJ \& Hustin J 1991 a Development of the early placenta: a morphometric study. Placenta 12 269-276.

Jauniaux E, Jurkovic D \& Campbell S 1991b In vivo investigations of anatomy and physiology of early human placental circulations. Ultrasound in Obstetrics and Gynecology $1435-445$.

Jauniaux E, Watson AL, Hempstock J, Bao Y-P, Skepper JN \& Burton GJ 2000 Onset of maternal arterial bloodflow and placental oxidative stress; a possible factor in human early pregnancy failure. American Journal of Pathology 157 2111-2122.

Jauniaux E, Hempstock J, Greenwold N \& Burton GJ 2003 Trophoblastic oxidative stress in relation to temporal and regional differences in maternal placental blood flow in normal and abnormal early pregnancies. American Journal of Pathology 162 115-125.

Jirkovska M, Janacek J, Kalab J \& Kubinova L 2008 Three-dimensional arrangement of the capillary bed and its relationship to microrheology in the terminal villi of normal term placenta. Placenta 29 892-897.

Jones CJP \& Fox H 1991 Ultrastructure of the normal human placenta. Electron Microscopy Reviews 4 129-178.

Kadyrov M, Kosanke G, Kingdom J \& Kaufmann P 1998 Increased fetoplacental angiogenesis during first trimester in anaemic women. Lancet 352 1747-1749.

Karimu AL \& Burton GJ 1994 The effects of maternal vascular pressure on the dimensions of the placental capillaries. British Journal of Obstetrics and Gynaecology 101 57-63.

Kaufmann P, Bruns U, Leiser R, Luckhardt M \& Winterhager E 1985 The fetal vascularisation of term placental villi. II. Intermediate and terminal villi. Anatomy and Embryology 173 203-214.

Kaufmann P, Luckhardt M \& Leiser R 1988 Three-dimensional representation of the fetal vessel system in the human placenta. Trophoblast Research 3 113-137.

Kaufmann P, Mayhew TM \& Charnock Jones DS 2004 Aspects of human fetoplacental vasculogenesis and angiogenesis. II. Changes during normal pregnancy. Placenta 25 114-126.

Kingdom JCP \& Kaufmann P 1997 Oxygen and placental villous development: origins of fetal hypoxia. Placenta 18 613-621.

Kuzmina IY, Hubina-Vakulik GI \& Burton GJ 2005 Placental morphometry and Doppler flow velocimetry in cases of chronic human fetal hypoxia. European Journal of Obstetrics, Gynecology, and Reproductive Biology 120 139-145.

Leach L 2002 The phenotype of the human materno-fetal endothelial barrier: molecular occupancy of paracellular junctions dictate permeability and angiogenic plasticity. Journal of Anatomy 200 599-606.

Leach L, Lammiman MJ, Babawale MO, Hobson SA, Bromilou B, Lovat S \& Simmonds MJ 2000 Molecular organization of tight and adherens junctions in the human placental vascular tree. Placenta 21 547-557.

Leach L, Babawale MO, Anderson M \& Lammiman M 2002 Vasculogenesis, angiogenesis and the molecular organisation of endothelial junctions in the early human placenta. Journal of Vascular Research 39 246-259.

Li B, Sharpe EE, Maupin AB, Teleron AA, Pyle AL, Carmeliet P \& Young PP 2006 VEGF and PIGF promote adult vasculogenesis by enhancing EPC recruitment and vessel formation at the site of tumor neovascularization. FASEB Journal 20 1495-1497.
Mayhew TM 2002 Fetoplacental angiogenesis during gestation is biphasic, longitudinal and occurs by proliferation and remodelling of vascular endothelial cells. Placenta 23 742-750.

Mayhew TM, Charnock Jones DS \& Kaufmann P 2004 Aspects of human fetoplacental vasculogenesis and angiogenesis. III. Changes in complicated pregnancies. Placenta 25 127-139.

Pijnenborg R, Vercruysse L \& Hanssens M 2006 The uterine spiral arteries in human pregnancy: facts and controversies. Placenta 27 939-958.

Ray PS, Jia J, Yao P, Majumder M, Hatzoglou M \& Fox PL 2009 A stressresponsive RNA switch regulates VEGFA expression. Nature 457 915-919.

Reshetnikova OS, Burton GJ, Milovanov AP \& Fokin El 1996 Increased incidence of placental chorangioma in high altitude pregnancies; hypobaric hypoxia as a possible aetiological factor. American Journal of Obstetrics and Gynecology 174 557-561.

Seval Y, Sati L, Celik-Ozenci C, Taskin O \& Demir R 2008 The distribution of angiopoietin-1, angiopoietin-2 and their receptors tie-1 and tie-2 in the very early human placenta. Placenta 29 809-815.

Shalaby F, Rossant J, Yamaguchi TP, Gertsenstein M, Wu XF, Breitman ML \& Schuh AC 1995 Failure of blood-island formation and vasculogenesis in Flk-1-deficient mice. Nature 376 62-66.

Shiu YT, Weiss JA, Hoying JB, Iwamoto MN, Joung IS \& Quam CT 2005 The role of mechanical stresses in angiogenesis. Critical Reviews in Biomedical Engineering 33 431-510.

Soothill PW, Nicolaides KH, Rodeck CH \& Campbell S 1986 Effect of gestational age on fetal and intervillous blood gas and acid-base values in human pregnancy. Fetal Therapy 1 168-175.

Tertemiz F, Kayisli UA, Arici A \& Demir R 2005 Apoptosis contributes to vascular lumen formation and vascular branching in human placental vasculogenesis. Biology of Reproduction 72 727-735.

te Velde EA, Exalto N, Hesseling P \& van der Linden HC 1997 First trimester development of human chorionic villous vascularization studied with CD34 immunohistochemistry. Human Reproduction 12 1577-1581.

Wheeler T, Elcock CL \& Anthony FW 1995 Angiogenesis and the placental environment. Placenta 16 289-296.

Zhang EG, Smith SK, Baker PN \& Charnock-Jones DS 2001 The regulation and localization of angioopoietin-1, -2, and their receptor Tie2 in normal and pathologic human placentae. Molecular Medicine 7 $624-635$.

Zhang EC, Burton GJ, Smith SK \& Charnock-Jones DS 2002 Placental vessel adaptation during gestation and to high altitude: changes in diameter and perivascular cell coverage. Placenta 23 751-762.

Ziche M, Maglione D, Ribatti D, Morbidelli L, Lago CT, Battisti M, Paoletti I, Barra A, Tucci M, Parise G et al. 1997 Placenta growth factor-1 is chemotactic, mitogenic, and angiogenic. Laboratory Investigation $\mathbf{7 6}$ $517-531$.

Received 7 March 2009

First decision 15 April 2009

Accepted 21 May 2009 